vol. 29 - $n^{\circ} 4$ | 2013

Numéro ouvert

\title{
Partir, rester, habiter : le projet migratoire dans la littérature exilaire
}

Leave, Stay, Live: Migration Project in the Literature of Exile

Irse, quedarse, vivir: el proyecto migratorio en la literatura del exilio

\section{Constance De Gourcy}

\section{QpenEdition}

Journals

\section{Édition électronique}

URL : https://journals.openedition.org/remi/6631

DOI : 10.4000/remi.6631

ISSN : $1777-5418$

Éditeur

Université de Poitiers

Édition imprimée

Date de publication : 1 décembre 2013

Pagination : 43-57

ISBN : 979-10-90426-10-8

ISSN : 0765-0752

\section{Référence électronique}

Constance De Gourcy, «Partir, rester, habiter : le projet migratoire dans la littérature exilaire », Revue européenne des migrations internationales [En ligne], vol. 29 - n4 | 2013, mis en ligne le 01 décembre 2016, consulté le 14 avril 2022. URL : http://journals.openedition.org/remi/6631 ; DOI : https://doi.org/ 10.4000/remi.6631 


\section{Partir, rester, habiter: le projet migratoire dans la littérature exilaire}

\section{Constance De Gourcy ${ }^{1}$}

"Lorsque je voyage dans ma chambre, je parcours rarement une ligne droite : je vais de ma table vers un tableau qui est placé dans un coin ; de là je pars obliquement pour aller à la porte ; mais, quoique en partant mon intention soit bien de m'y rendre, si je rencontre mon fauteuil en chemin, je ne fais pas de façon, et je m'y arrange tout de suite" (de Maistre, [1794] 2002).

Prenant acte de la globalisation de la question migratoire ${ }^{2}$, de nombreux travaux privilégient désormais une approche centrée sur la diversité des figures migratoires et des contextes de migration. Révisant les explications classiques, ces nouvelles orientations de recherches accordent une place centrale aux notions de stratégie, d'autonomie et de projet (Ma Mung, 2009 ; Berthomière, 2009 ; Bordes-Benayoun et Schnapper, 2008 : 72). Bien qu'elles soient porteuses d'un horizon de sens qui tienne compte de l'importance de l'action sur les structures, ces notions nécessitent d'être interrogées à l'aune des effets implicites qu'elles véhiculent tant sur le plan analytique que sur le plan méthodologique. Dans quelle mesure, en effet, celles-ci impliquent-elles une conception orientée de l'acteur migrant, conception qui privilégierait la capacité à agir de l'acteur indépendamment des contraintes qui pèsent sur l'action ? Aussi, dans le cadre de cet article, nous souhaitons proposer une réflexion théorique et méthodologique sur les usages de la notion de projet migratoire afin d'en préciser ses apports et ses limites.

Pour ce faire, le projet migratoire sera considéré comme un outil descriptif et analytique des modulations liées au vécu dans le pays d'installation. Derrière la réalité des chiffres de l'immigration, les individus sont pris dans des configurations sociales, politiques, familiales et, plus largement, relationnelles qui influent sur le projet, sa temporalité et le modulent dans un sens ou dans un autre. L'événement biographique, I'aléa, la rencontre deviennent-ils, grâce à

1 Aix-Marseille Université, CNRS, LAMES (7305), 13094 Aix-en-Provence Cedex 2 ; constance.degourcy@univ-amu.fr

2 Comme le souligne Catherine Wihtol de Wenden : « Le volume des migrations a triplé en trente ans et, presque toutes les régions du monde sont aujourd'hui concernées par le départ, le transit ou l'accueil de populations de plus en plus mobiles, aux profils de plus en plus diversifiés " (Wihtol de Wenden, 2009: 15). 
l'introduction de cette notion, la matière première permettant de préciser le sens d'un mouvement afin de retrouver, au-delà du poids des chiffres sur les migrations, la texture des vécus et des dynamiques qui les sous-tendent ? II reste que poser cette question revient à modifier la perspective : il ne s'agit plus tant, en effet, de considérer des individus avec des caractéristiques sociales données qui imposent leurs marques au vécu, que de voir comment ce vécu et les contraintes de toutes sortes que rencontrent les migrants modifient/infléchissent les parcours et requièrent de leur part des réponses inventives, inédites, qui peuvent entrer en concordance (ou pas) avec leurs appartenances sociales, professionnelles et culturelles. Certes, cette perspective n'est pas entièrement nouvelle puisqu'elle consiste à s'intéresser, selon la célèbre formule deThomas, à la manière dont les individus définissent la situation, mais nous souhaitons en pousser la logique jusqu'au bout : celle qui consiste dès lors à prendre en compte la façon dont le contexte agit sur la définition de la situation et conduit le migrant à (re)définir le sens de son parcours.

Pour étayer cette réflexion, nous partirons d'un corpus constitué d'ouvrages issus de la littérature exilaire ${ }^{3}$. Si la littérature capte les moments et les événements les plus ténus liés à la décision de migrer et à la mise en œuvre de la migration, elle n'est pas qu'un " réservoir d'histoires ou une façon de les raconter, c'est une manière de construire le monde même où des histoires peuvent arriver, des événements s'enchaîner, des apparences se déployer " (Rancière, 2011 : 19). Elle apparaît ainsi comme un poste privilégié pour observer et analyser la place que les parcours et expériences migratoires occupent dans la fabrique contemporaine d'un imaginaire de la migration et saisir la façon dont ces problématiques raisonnent/résonnent dans des sociétés de plus en plus traversées par la question de l'altérité. Analyser le projet migratoire sous l'angle littéraire offre en outre un point de vue diversifié et surplombant des différentes modalités de constitution et d'accomplissement d'un parcours sans préjuger des raisons pour lesquelles il est mis en œuvre.

Afin d'étudier cette dynamique à l'œuvre dans l'élaboration des projets de départ, nous nous attacherons, d'abord, à préciser les enjeux théoriques sous-jacents à cette notion. Nous formulerons ensuite quelques propositions méthodologiques permettant d'en définir et d'en préciser les conditions d'observations avant de considérer les implications du projet comme outil de saisie du mouvement. Au final, nous souhaitons montrer combien le recours à cette notion se révèle heuristique pour réintégrer dans l'analyse des éléments contextuels dont la portée explicative est rarement prise en compte. C'est à cette condition qu'il devient possible d'envisager un parcours migratoire comme une activité orientée, dont le sens n'est pas donné a priori mais se constitue dans l'interaction avec l'environnement ${ }^{4}$ dans lequel se meut l'individu.

\footnotetext{
3 Nous parlerons de "littérature exilaire " au sens large, en entendant par ce terme les récits qui font de l'exil et des migrations la matière première de leur investigation. Ce choix s'inscrit dans la lignée des travaux qui proposent de considérer le roman comme un laboratoire d'analyse du social à partir des catégories nouvelles qu'il offre à I'analyse sociologique (Barrère et Martuccelli, 2009).

4 Nous entendons ce terme au sens physique mais aussi social et culturel.
} 


\section{Le projet migratoire : du renouveau au renouvellement des approches}

Si la notion de " projet migratoire " semble connaître depuis les années 1970 un succès grandissant dans la littérature française et anglo-saxonne sur les migrations, ce constat ne saurait masquer l'intérêt de connaissance qu'il sert (Habermas, 1979) ou plus largement les logiques sociales qui concourent à son recours. L'alternative - mais s'agit-il d'une alternative ? - pourrait se résumer ainsi : faut-il historiciser la notion de projet migratoire et l'inscrire dans un contexte précis lié au développement de migrations centrées sur un projet individue $\mathrm{I}^{5}$ ou la considérer comme une notion susceptible de réviser les travaux antérieurs à l'aune de ses vertus heuristiques ${ }^{6}$ ?

Avant d'aborder ces questions, il nous faut délimiter le champ d'observation des migrations pouvant faire l'objet d'un tel projet. En effet, toutes les migrations, comme les migrations d'exil qui répondent aux pratiques antiques de l'ostracisme ou les migrations de réfugiés plus récentes, ne peuvent faire l'objet $d^{\prime}$ 'une lecture en termes de projet migratoire ${ }^{7}$. En revanche, la question se pose pour le cas des expatriés et des immigrés pour lesquels le déplacement n'est pas nécessairement contraint ou imposé. Les premiers comme les seconds choisissent le plus souvent de vivre dans un pays étranger même si le statut de la migration peut paraître ambigu et les conditions du choix restreintes. Comme le souligne Edward Said (2008: 250) " les officiers coloniaux, les missionnaires, les experts techniques, les mercenaires et les conseillers militaires en mission, vivent en exil, mais ils n'ont pas été bannis ". Une analyse en termes de projet migratoire permet également de repenser la corrélation souvent (trop) rapidement établie entre pauvreté et misère d'une part et émigration d'autre part. Ainsi les migrations du Sud vers le Nord peuvent-elles se comprendre et s'expliquer sous de nouveaux angles : ce sont moins les raisons économiques qui mettent les candidats au départ sur les routes de l'exil que la perspective de " rupture initiatique dans l'optique d'un futur retour glorieux permettant d'obtenir la reconnaissance sociale de son groupe et d'accéder à une véritable majorité sociale " (Timera, 2001).

Cette présentation non exhaustive des contextes de départ rappelle que les conditions de mise en œuvre de la migration supposent un minimum d'autonomie dans la définition d'un projet migratoire. Pour autant, ce positionnement ne saurait se réduire à l'opposition entre déterminisme et liberté : adopter le point de vue de l'autonomie ne signifie pas que les migrants évoluent dans un univers sans contraintes ni entraves, un univers de libre choix. Les contraintes et

5 Projet individuel qui apparaît en germe dans ce qu'Abdelmalek Sayad a pu appeler le " troisième âge de l'immigration ".

6 On se réfère ici à I'intervention de Scioldo-Zürcher Yann (2011) Stratégies des acteurs migrants et projet migratoire, Atelier de recherche Stratégies des acteurs migrants et projet migratoire, atelier coordonné par Mohammed Charef et William Berthomière, Faculté Polydisciplinaire Taroudant, 15-16 juin 2011.

7 Mentionnons cependant à titre d'exception le travail mené par Michalon Bénédicte (2012) Enfermement et projet migratoire. Les trajectoires d'étrangers en rétention en Roumanie, Le projet migratoire: intentionnalité et expériences des acteurs, Quelles approches méthodologiques ?, séminaire coordonné par Florence Boyer et William Berthomière, juillet 2012. 
épreuves marquent bien les migrations autonomiques (De Gourcy, 2005), mais leur statut explicatif diffère de celui accordé dans les migrations d'exil et/ou de réfugié. Dans cet écart, qui fait saillir diverses expériences migratoires, se joue le statut de la migration et ses effets pour les migrants et leurs groupes. Le projet migratoire apparaît ainsi comme un révélateur du rapport que les individus entretiennent à l'égard de la migration et des possibles dont elle est investie. II se manifeste également comme une ligne de partage entre les migrations liées aux déplacements subis, contraints (et vécus comme tels par celui ou celle exilé.e ou réfugié.e - qui les met en œuvre) et les migrations où la contrainte fait place à l'obligation personnelle dont se dote le migrant pour donner une habitabilité au monde dans lequel il se meut ${ }^{8}$.

En tenant compte de ces quelques éléments de clarification, nous pouvons, à présent, répondre à la question soulevée précédemment en tentant de concilier les deux termes de l'alternative présentée. En effet, si la notion de projet migratoire émerge en France dans le contexte des années 1970, après la fin des flux générés par l'appel étatique à la main-d'œuvre internationale, celle-ci apparaissait déjà en filigrane dans de nombreuses approches théoriques (Rosental, 1999). En témoignent ainsi les travaux qui effectuent une relecture des travaux pionniers ${ }^{9}$ à l'aune de cette notion. Ce recours implicite souligne l'intérêt heuristique de la notion tant sur le plan méthodologique que sur le plan théorique. Considérant sa visée méthodologique, la notion permet ainsi de prendre en compte les aspirations individuelles à la migration et de les inscrire dans une perspective sociétale, économique, politique ou autre si bien qu'une analyse en termes de projet migratoire permettrait, en fonction des données disponibles, de nuancer et/ou de relativiser des approches centrées sur les déterminants de la migration (De Gourcy, 2005). Sur le plan théorique, la notion se présente comme relevant du schème actanciel lequel implique la " reconnaissance de l'intentionnalité de I'action, son irréductibilité à une détermination causale " (Berthelot, 1998 : 76). C'est donc à une tout autre conception de l'individu migrant et de la migration que nous conduit cette notion, une conception qui fait de l'intentionnalité et, pourrait-on ajouter, de l'intentionnalité en acte, la matière même des études se revendiquant d'une telle approche. Pour autant se pose la question de savoir où commence l'intentionnalité. À quelle étape du procès migratoire se manifeste-t-elle ? Comment saisir l'intention dès lors que l'action migratoire $n^{\prime}$ est pas réalisée ${ }^{10}$ ? Peut-on ainsi considérer, en l'absence de contraintes liées à la mise en œuvre de la migration (ou de ses éventuels déterminants), que la

8 En ce sens, la mobilité n'est pas ce qui met fin à l'expérience de l'habiter mais ce qui la rend possible.

9 Ainsi, les trois âges de l'immigration développés par Sayad peuvent-ils se prêter à une lecture en termes de projet migratoire ; le changement d'âge se traduisant par un changement de projet ? Voir Timera Mahamet (2012) Du projet à I'analyse de la configuration sociale de l'acteur migrant, Le projet migratoire : intentionnalité et expériences des acteurs, Quelles approches méthodologiques?, séminaire coordonné par Florence Boyer et William Berthomière, juillet 2012.

10 De ce point de vue l'imaginaire constitue une entrée possible pour analyser la formation du projet migratoire indépendamment des conditions de sa mise en œuvre (Fouquet, 2007). 
décision de partir constitue le seuil liminal du projet ${ }^{11}$ ? II nous reste à préciser ces questions en tentant d'expliciter les conditions d'observation du projet migratoire.

\section{Observer le projet migratoire : questions de méthode}

Définir les conditions d'observation du projet suppose préalablement de savoir à quelle étape du procès migratoire l'observer. Cette question, simple en apparence, se complique dès lors que l'on considère avec Paul-André Rosental (1999 : 207) que " plutôt que de résulter exclusivement de stimuli extérieurs et immédiats, la décision de partir ponctue des mécanismes de maturation souvent longs, que nous avons qualifiés de "projets migratoires" ". Cependant, cette approche ne permet pas de distinguer ce qui relève d'un processus individuel ou collectif d'élaboration du projet des conditions de sa mise en œuvre, lesquelles s'inscrivent dans une temporalité administrative différente. Aussi, nous proposons de distinguer dans l'élaboration du projet deux phases qui sont le résultat d'un processus interactif mais qui, sur le plan méthodologique, se laissent appréhender différemment :

- La phase de définition, phase d'une durée variable au cours de laquelle le candidat - I'aspirant au départ - inscrit la migration dans un projet révélateur

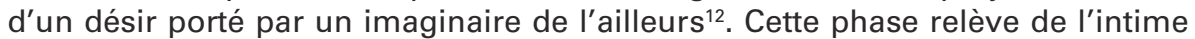
ou du privé : le projet n'est pas encore publiquement énoncé, il apparaît comme un " possible " à réaliser dans un futur plus ou moins proche et participe à ce titre de l'élargissement de l'horizon d'attentes de l'individu. Sur le plan méthodologique, cette étape n'est pas observable en tant que telle ; elle n'existe qu'à travers le filtre de ce qu'en dira (ou pas) le locuteur ${ }^{13}$. Nous y reviendrons ultérieurement.

11 La citation suivante extraite du célèbre Paysan polonais illustre ainsi un cas limite de compréhension de la mise en mouvement à partir de la notion de projet. Précisons toutefois que le voyage dont il est question s'effectuera sur une courte distance mais, replacé dans l'itinéraire biographique de l'individu, il devient significatif : " Dès le départ de notre frère Alesky, j'ai commencé à préparer mon voyage bien que ne sachant pas où aller. Je savais que je ne retournerai pas chez M. Degurski, mais je n'avais aucun projet précis. Je suis parti à trois heures [...]. J'avais préparé un petit baluchon que j'avais caché dans l'étable où je I'ai retrouvé après avoir dit au revoir à tout le monde à la maison. Je I'ai pris et je suis parti à I'aventure, sans but " (Thomas et Znaniecki, 1998 : 143).

12 Dans les entretiens menés auprès de migrants comme dans la littérature exilaire, on retrouve ces notions de désir et d'imaginaire comme activateur d'une ouverture des possibles. Toutes les destinations lointaines faisant l'objet de ce type d'investissement ne donneront pas lieu à une migration mais, lorsque c'est le cas, le projet migratoire s'ancre dans une géographie symbolique: "C'est à Boston, du reste, dans un petit café orange avec deux tables à la terrasse, le French Café, qu'est né mon désir de voir Paris " (Huston et Sebbar, $1986: 88$ ) ; "Les visites estivales des Américains furent vraisemblablement la cause première pour laquelle j'imaginais, adolescent, que j'émigrerais plus tard outreAtlantique " (Sebald, $1999: 84)$.

13 Si nous reprenons la citation extraite du Paysan polonais, on peut ainsi supposer que si le paysan polonais mentionne ce voyage, c'est parce que dans le récit qu'il propose rétrospectivement de son parcours, celui-ci apparaît comme une première étape d'un procès que viendra clore la migration vers l'Amérique. Commentant son récit, les deux auteurs parlent de " disposition préexistante au changement et un désir d'aventure " (Thomas et Znaniecki, 1998 : 143). 
- La phase qui précède le départ, phase consacrée à sa préparation. C'est lors de cette étape que le candidat formalise son projet, I'annonce à ses proches, engage les formalités nécessaires sur le plan matériel, administratif, etc. II en résulte une série d'actes et de traces observables liés à l'accomplissement des démarches à mettre en œuvre avant le départ (demandes ou renouvellement de visa, constitution d'un pécule financier, activation des réseaux pour le départ et/ ou l'accueil dans le pays d'arrivée, etc.) ; la plupart de ces actes peut s'agréger dans des statistiques de départ.

La migration ne résulte donc jamais d'une démarche spontanée ou improvisée, car le départ vient ponctuer une phase souvent longue de préparation. $C^{\prime}$ est lors de cette phase de préparation que s'observent également les conséquences du projet sur les parcours biographiques, car partir entraîne souvent l'adoption d'un style de vie (Weber, 1964) qui anticipe l'absence et ses effets sur les liens sociaux. Cette phase apparaît enfin comme le moment décisif où se construisent les qualités d'hospitalité du pays visé lesquelles, en retour, contribuent à l'élaboration du projet et à sa définition.

Au cours de cette phase, les candidats à l'émigration s'ouvrent à l'expérience de ceux déjà partis et cherchent à obtenir, sur le mode de l'enquête, des informations relatives au pays d'accueil. Les échanges épistolaires entre les migrants et les individus restés sur place se sont longtemps fait l'écho de ces demandes d'informations. Aujourd'hui avec les technologies de l'information et de la communication (TIC) s'inventent de nouveaux régimes d'exposition de soi (Diminescu, 2007) qui réintroduisent le quotidien au cœur de l'expérience migratoire. Les interactions désormais possibles entre personnes inconnues sur la base des similitudes - de sexe, de génération, de nationalité ou de statut, etc. -, qu'elles sont supposées avoir en commun, génèrent de nouvelles requêtes par le biais de sites Internet. Dans une enquête menée sur le départ pour études d'étudiants algériens, nous avons montré comment ces échanges participaient d'une forme d'évaluation du projet : dans un contexte où les migrants ne se déplacent pas, ou plus, vers des collectifs de sédentaires mais vers des collectifs où l'inconnu est une figure constitutive de l'être urbain (Sennett, 1995), il s'agissait pour ces candidats à l'émigration de voir comment la requête allait être accueillie, perçue par les étudiants déjà sur place (De Gourcy, 2007). Se déploient ainsi, par le biais de ces requêtes, de véritables tactiques sur les lieux de l'autre (de Certeau, 1980) où I'enjeu consiste moins à être perçu comme un "étranger " que comme un " inconnu ", soit comme un étudiant parmi d'autres.

En somme, les démarches consacrées aux différentes formalités nécessitent d'être considérées indépendamment de la migration, car elles ne préjugent pas de l'effectivité du départ. II n'est en effet pas certain que le candidat mette en œuvre la migration à l'issue d'une phase de préparation, soit parce que celle-ci n'aura pu, sur le plan des formalités administratives, être validée, soit parce que d'autres projets concurrents ont pu prendre le pas sur la migration ${ }^{14}$. Que le départ ait lieu ou pas, l'analyse du projet migratoire s'ouvre en effet à

14 Ainsi, il n'est pas rare de constater chez les jeunes adultes étudiants enquêtés que la rencontre amoureuse constitue un événement biographique venant mettre un terme au projet migratoire. On peut se rapporter sur le sujet à l'ouvrage de Vito Teti (2011) qui propose une anthropologie du "rester". 
d'autres éventualités comme les possibilités de renoncement au projet ou de report ( $\mathrm{y}$ compris plusieurs générations après une première migration), etc. qui peuvent faire l'objet d'une analyse à part entière. Ainsi, ce que I'on appelle aujourd'hui le "tourisme des racines " peut-il être vécu, de la part des descendants de migrants et/ou d'exilés, comme une forme de retour - retour différé, " posthume " accompli de façon ponctuelle - sur les lieux de l' " origine " et sur les traces des ascendants.

Considérer les phases de définition et de préparation du projet indépendamment de la séquence du départ permet également de les extraire d'une temporalité linéaire et progressive ${ }^{15}$ : ces phases peuvent se reproduire et se réactiver après la migration, dans le pays d'accueil. Tout se passe en effet comme si le processus qui participe de l'élaboration du projet se reproduisait à différentes étapes du procès migratoire. La période correspondant à l'installation dans un pays d'accueil peut ainsi être considérée comme une phase dynamique, phase au cours de laquelle de nouveaux projets verront le jour (y compris celui de rester et de s'installer durablement). La qualification d'une étape, comme étant une étape de transit ou d'installation durable, se révèle, dans ce constant ajustement entre attentes et révisions d'attentes, au regard de ce que d'autres pays, d'autres destinations laissent supposer en termes d'ouverture au projet. Comme le souligne Emmanuel Ma Mung, le " projet migratoire est sans cesse alimenté par l'acte migratoire " (Ma Mung, 2009) et de cette dynamique résulte les conditions d'une redéfinition en fonction du vécu et des opportunités qui s'offrent à eux.

Observer le projet migratoire suppose ainsi de penser les deux phases dans leur rapport avec le vécu sur place, dans le pays de départ, comme dans le pays d'installation. Pour les étudiants algériens résidant en France évoqués précédemment, le désir d'ailleurs - majoritairement le Québec - se forge ainsi progressivement à l'épreuve du vécu dans le pays d'installation et sur la base d'un retour en Algérie perçu, au fur et à mesure, comme impossible, si ce n'est sous une forme ponctuelle lors de courts séjours. Aussi, importe-t-il à présent de revenir sur la phase de (re)définition du projet en essayant de comprendre quels en sont les mécanismes déclencheurs.

\section{La phase de (re)définition: les sources et ressources du projet}

À la différence de la phase de préparation, phase observable à partir de traces matérielles et de récits de migrants, celle consacrée à la définition/redéfinition du projet s'observe rétrospectivement à partir des seuls récits que font ceux qui s'apprêtent à partir (primo-migrants) ou ceux, qui après avoir vécu l'immigration dans un pays d'accueil, envisagent un nouveau départ. Dans les deux cas, l'élaboration du projet ou la formation d'une nouvelle étape tiennent à la confrontation/comparaison entre ce que les migrants imaginent de l'ailleurs,

15 Si la temporalité du projet migratoire se révèle dans ce rapport d'ajustement constant entre projet et vécu sur place, on notera que tout autre est la temporalité administrative qui régule le séjour du migrant par le biais de visas et/ou de cartes de séjour. Ceux-ci soumettent la temporalité de l'installation et le rythme de l'habitant à une durée contrainte, administrativement définie. 
ou avaient imaginé, anticipé, et le vécu sur place. Ces récits comparatifs révèlent en creux l'imaginaire qui a contribué ou contribue à nouveau à le façonner.

Par la comparaison s'opère en effet une révision des savoirs fondée sur le décalage entre ce qui a été dit d'un pays et ce qui en est perçu ou vécu. Les situations vécues dans le pays d'origine ou d'installation se présentent tout à la fois comme partagées et idiosyncrasiques. Partagées lorsqu'elles donnent prises sur des éléments entendus ou racontés lesquels peuvent, en retour, fonder le sentiment d'une appartenance à une même " condition " (NDdiaye, 2007) liée à l'expérience vécue en tant qu'étranger. Elles peuvent également être idiosyncrasiques dès lors que les situations rencontrées par le migrant témoignent d'un décalage par rapport au cours " normal " des choses.

Dans les deux cas, c'est par la médiation des situations vécues que se forme une représentation sur I' " atmosphère " et I' " ambiance " (Barrère et Martuccelli, 2009) des pays dans lesquels évoluent les migrants et que peut se forger à partir de cette représentation un (nouveau) projet de départ.

Dans la littérature, les déclencheurs du projet sont étroitement liés au ressenti des personnages, à leur appréciation qualitative de ce qu'ils sont amenés à vivre au quotidien, un quotidien perçu souvent comme monotone. L'individu migrant tel qu'il est mis en scène dans la littérature apparaît ainsi comme maître de ses déplacements dans un monde où les frontières sont moins figurées spatialement qu'associées à des espaces à qui sont attribuées des qualités d'hospitalité différentes. Dans l'extrait littéraire suivant, la comparaison entre deux " ambiances ", I'une propre au pays d'origine (I'Allemagne) et l'autre à celui d'accueil (I'Égypte) témoigne, à l'avantage du second, de l'ouverture du champ des possibles dont il est investi. L'ennui, comme marqueur d'ambiance dans le pays d'origine, apparaît en effet comme un mécanisme déclencheur dans la décision du personnage de partir s'installer ailleurs dans un pays découvert lors d'un voyage :

" Je demandais à Jutta ce qu'elle faisait en Égypte et elle me répondit en riant: - Oh, c'est une longue histoire ! Je suis venue en voyage organisé et le pays m'a tellement plu que cela m'a gâché la vie ensuite. Lorsque je suis revenue en Allemagne, tout m'a semblé ennuyeux. Alors j'ai décidé de venir me fixer ici et m'y voilà maintenant »

(El Aswani, 2009 : 110).

Ces marqueurs $d^{\prime}$ ambiance et $d^{\prime}$ atmosphère à partir desquels se forgent des représentations des espaces vécus apparaissent soit comme des déclencheurs du projet, comme dans l'extrait précédent, soit comme ce qui justifie de nouveaux départs dans l'itinéraire biographique du migrant. En effet, le vécu sur place est propice à de nouvelles comparaisons avec ce que laissent entrevoir d'autres destinations. Cette tension entre des attentes qui n'ont pu pleinement s'accomplir et l'ouverture que laissent deviner les nouveaux récits d'ailleurs peut participer de la redéfinition du projet et enclencher la séquence liée à la préparation d'un nouveau départ, migration de retour ou d'installation dans un nouveau pays. Ce moment de transition et de (re)mise en mouvement est souvent décrit, dans les récits littéraires, à partir des impressions ressenties. $C^{\prime}$ est donc moins la contrainte qui prédomine dans ce type de migration que la nécessité de trouver dans l'ailleurs un cadre propice à l'épanouissement de soi : 
" J'avais passé près de trois années à Manchester quand, ayant terminé mes travaux de recherche à l'été 1969 , je quittais la ville pour réaliser un projet que je caressais depuis assez longtemps et aller enseigner en Suisse. Bien que, revenant d'une Manchester noire de suie et en complète décrépitude, je fusse profondément touché par la beauté et la variété des paysages suisses [...], pour diverses raisons, tenant au mode de vie local ou à ma condition de pédagogue, j'ai eu tôt fait de ne plus me sentir bien dans ce pays. II ne s'était pas écoulé une année que je décidais de retourner en Angleterre "

(Sebald, 1999 : 207-208).

Au cours de cette phase de définition et redéfinition du projet affleurent également les silences et les non-dits de la migration dont Sayad (1999) a pu dire, concernant l'émigration algérienne, qu'ils participaient de la nécessité de dissimuler la réalité de l'exil afin de perpétrer le processus migratoire ${ }^{16}$. Dans le cas des étudiants algériens qui poursuivent leurs études en France, une connaissance par expérience s'élabore progressivement à partir de situations perçues comme révélatrices des tensions qu'ont pu vivre leurs grands-pères ou leurs proches partis travailler comme main-d'œuvre en France. Lors de cette actualisation du passé à partir du présent, les non-dits de la migration, les silences se reconstituent progressivement.

La mémoire constitue ainsi le fil conducteur, la médiation permettant de retrouver ce que I'on n'a soi-même pas vécu, mais qui avait pu donner une tonalité sensible d'ensemble - une ambiance - aux faits racontés. Le partage de l'expérience entre ces générations de migrants s'opère ici sur la base de souvenirs partagés, souvenirs que nous définirons moins comme la combinaison en une seule version des "différents points de vue de ceux qui se rappellent un événement donné " (Margalit, 2006) que comme la reconstitution de la mémoire à partir de l'oubli provisoire ${ }^{17} \mathrm{~d}^{\prime}$ une impression ou d'une tonalité donnée aux récits migratoires. L'expérience ainsi reconstituée par la médiation de la mémoire contribue à faire exister ce décalage entre des attentes souvent très fortes liées à la migration et le vécu sur place.

En somme, pour un individu donné, le séjour qui fait suite à une première migration ne vient pas nécessairement clore une séquence migratoire mais offre les conditions possibles pour réorienter ou redéployer le sens de ses placements et déplacements dans l'espace ${ }^{18}$. Ainsi, importe-t-il de considérer le projet migratoire comme un outil pour comprendre des enchaînements et la façon dont ils se combinent. Il permet en effet de saisir diverses " matérialités " telles que les événements, rencontres, les différentes " atmosphères " d'un

16 II est sans doute aussi possible de proposer I'hypothèse selon laquelle les secrets et dissimulations des travailleurs immigrés algériens à l'égard des concitoyens restés dans le pays d'origine (Sayad, 1999) participaient du maintien de l'objectif initial du projet migratoire - revenir au pays - alors même que cet objectif se révélait de plus en plus vain sauf, à revenir au pays aussi démunis que lorsque ces derniers l'ont quitté.

17 On notera que comme leurs aïeux immigrés, ces étudiants sélectionnent dans leurs récits oraux ou écrits ce qui peut (ou ne pas) être dit, contribuant ainsi à entretenir les illusions de l'immigré.

18 L'extrait suivant montre combien un séjour ponctuel, avec la certitude qu'il le serait, a progressivement laissé place à un " provisoire durable " : "As-tu eu une époque "touristique" au tout début, quand tu es venue vivre à Paris ? Moi qui ne pensais y rester qu'un an, je marchais partout, je visitais tous les quartiers, presque toutes les rues, persuadée que je ne les reverrais plus jamais " (Huston et Sebbar, 1986 : 35). 
lieu, etc., qui, à la jonction entre une histoire personnelle et la part d'imprévu et d'aléa liée à la découverte de nouveaux territoires et de leurs ressources, donnent lieu à des réorientations ou à des bifurcations de parcours alors même que celles-ci n'étaient pas planifiées ou anticipées au moment du départ. Pris comme tel, le projet migratoire apparaît donc davantage comme un outil de saisie du mouvement, ou de la (re)mise en mouvement, qu'un outil permettant de penser l'arrêt, la fixité. Précisons également qu'à cette mise en mouvement correspond une mise en sens par le projet migratoire grâce à la structure du récit ${ }^{19}$ qui permet de saisir différentes étapes sur le mode de l'enchaînement. Dans cette opération se joue le sens donné au projet par celui qui l'interprète. Ce sens étant lui-même étroitement lié aux théories implicites du sujet qui orientent cette interprétation.

\section{Le projet migratoire au prisme de l'habiter}

II nous reste à présent à examiner dans quelle mesure la mise en récit des séquences qui constituent le procès migratoire donne du sens - une orientation - au parcours des migrants, tant sur le plan biographique que sur le plan géographique. Autrement dit, les parcours migratoires ne s'interprètent pas uniquement comme le résultat de déplacements géographiques ; leur compréhension mobilise des éléments relatifs au contexte dans lequel évolue(nt) le ou les migrant(s). L'examen de la littérature exilaire apporte là encore des éléments de réponse, éléments sans doute incomplets mais présentés ici à titre exploratoire.

Si les sciences sociales ont longtemps mobilisé le registre de la rupture, du déracinement, du dépaysement pour qualifier l'expérience migrante, la littérature met en scène des parcours dans lesquels l'unité biographique se construit progressivement grâce à la migration. C'est donc moins l'expérience du déracinement qui fait l'objet d'un récit littéraire que l'expérience de l'habiter dans un espace perçu comme propice à la construction de nouvelles attaches, un espace dont les qualités d'hospitalité l'inscrivent dans la continuité ${ }^{20}$ du parcours accompli. Les mobilités et migrations que les personnages mettent en œuvre apparaissent ainsi comme des ressources offrant un recentrage possible sur d'autres repères géographiques que ceux liés aux espaces de l'origine souvent associés à la période de l'enfance et de la jeunesse.

Dans l'extrait littéraire suivant, un voyage accompli pendant les études universitaires est présenté comme l'élément déclencheur permettant au locuteur d'investir de nouveaux espaces de déploiement du chez-soi. Après avoir vécu et grandi en Amérique il a choisi de s'établir au Maroc :

19 Rappelons à la suite de Daniel Bertaux (1976) que le récit biographique est travaillé et construit selon un schéma préétabli qui est le schéma chronologique d'une vie. Or la chronologie est déjà une sélection qui fonctionne sur une temporalité linéaire.

20 Cette lecture implique donc de considérer que les ruptures et changements induits par les déplacements sont à resituer plus largement dans une histoire de vie dont la continuité est assurée par la mémoire. Comme le souligne le philosophe, "La mémoire [...] assure la continuité temporelle, en permettant de se déplacer sur l'axe du temps ; elle permet de se reconnaitre et de dire moi, mien " (Ricœur, $1995: 188$ ). 
"Chez ma mère. J'ai failli dire "chez moi", mais si ces mots existent toujours, ils ont perdu toute valeur. Chez moi, maintenant, c'est à Tanger. [...] II y a deux ans, je jouais dans l'équipe de Princeton; aujourd'hui, je vis dans un port d'Afrique du Nord en tentant d'écrire mon premier roman. Ma vie a changé du tout au tout. Le Pérou a enclenché ce processus ; Tanger le poursuit » (Hopkins, 2011 : 43).

Les évaluations réflexives et rétrospectives qu'accomplissent les personnages soulignent le lien fort établi entre déplacements géographiques et construction de soi dans un espace où il devient possible de se projeter. Dans l'extrait suivant, le locuteur se situe dans une temporalité, celle d'un devenir au regard des migrations accomplies, devenir qui amène à considérer le parcours comme une " matière " structurée par des rencontres et des opportunités ainsi que par des actes. Mais, l'anticipation se révèle aussi comme un accomplissement de la migration et de ses effets sur le parcours :

"C'est dans cette région dont les habitants sont réputés superstitieux, bornés et renfermés que nous avons choisi de nous enraciner. [...] notre fils est né sous les mains expertes d'une sage-femme berrichonne nommée Proust, et c'est peut-être dans le Berry que nos attaches sont maintenant les plus sereines et les plus sûres. [...] [C'est] là, enfin, dans cette terre française si secrète de bocages et d'étangs, de bouchures et de bois, de petites églises romanes et de vaches charolaises, que nous aurions envie de reposer à la fin de l'histoire " (Huston, 1999).

Le projet migratoire, cet outil d'analyse des fluctuations et des pulsations des parcours, ne permet-il pas, en ordonnant ces derniers selon des séquences précises, de saisir le sens d'une quête, celle qui consiste à rechercher, dans I'ailleurs, les conditions d'habitabilité d'un territoire donné ? Et si les espaces, les territorialités plurielles qui composent les espaces vécus, " configurent les actions, les interactions et les productions langagières "(Deprez, 2007), on ne peut que souligner combien, dans les deux derniers extraits présentés, la notion de " projet migratoire " butte désormais sur le sens d'un chez-soi reconstitué à partir de nouveaux modes de présence au monde et, lors de cette ultime évocation qui donne sens et finalité au parcours, perd toute nécessité. Aussi, pouvons-nous proposer de considérer l' " autonomie dans la migration " comme la possibilité de donner par la migration une habitabilité à l'espace traversé. Cette proposition s'oppose d'une part, aux théories fixistes de l'appartenance qui considèrent le lieu de naissance comme le lieu d'appartenance ${ }^{21}$ et, d'autre part, aux approches par les déterminants économiques lesquelles, en occultant le socle anthropologique de l'acte migratoire, le réduit à une conception utilitariste.

\footnotetext{
21 Voir à ce sujet I'analyse proposée sur les récits de fondation (Detienne, 2001). On peut aussi trouver une résonance littéraire de ce thème dans I'œuvre d'Amin Maalouf (1998) : "Mon but n'étant pas - on I'aura compris - de retrouver en moi-même une quelconque appartenance "essentielle" dans laquelle je puisse me reconnaître, c'est l'attitude inverse que j'adopte : je fouille ma mémoire pour débusquer le plus grand nombre d'éléments de mon identité, je les assemble, je les aligne, je n'en renie aucun ".
} 


\section{Conclusion}

Nous avons tenté de proposer une réflexion sur les apports et les limites de la notion de projet migratoire à travers le regard qu'offre la littérature sur ce sujet. Dans cette perspective, nous avons été amenés, en nous appuyant sur un corpus d'œuvres littéraires, à proposer quelques pistes théoriques et méthodologiques permettant de préciser ses usages. La littérature constitue de ce point de vue un poste d'observation privilégié de la façon dont des personnages sont (re)mis en mouvement par la migration et, comment par ces dé-placements successifs ils s'approprient des espaces et des mondes sociaux nouveaux, différents de ceux expérimentés précédemment. On notera à ce sujet l'effacement des migrations de nécessité ou liées à des mobiles économiques derrière des migrations mises en œuvre pour les possibilités d'ouverture et/ou les potentialités émancipatrices qu'elles recèlent. Pour autant, ces migrations ne sont pas animées par des mobiles hédonistes, elles répondent plus largement à la nécessité de repenser I'ancrage dans la mobilité et ce faisant dessinent un monde ouvert, fluide, un monde où le "droit à la mobilité " est une condition du vivre-ensemble.

Ce bilan serait cependant incomplet sans l'intégration de la notion de voyage dans la compréhension des séquences migratoires et de l'évaluation rétrospective que fait le locuteur de son parcours. Rencontré à plusieurs reprises dans la littérature exilaire ${ }^{22}$, le voyage - cette expérience au cours de laquelle se cristallise un imaginaire et des schèmes d'attractivité du lieu - apparaît comme une étape décisive dans l'élaboration du projet migratoire et dans sa redéfinition. Certes, la notion de projet migratoire se présente, comme l'avait souligné Paul André Rosental, comme " la résultante de séquences génératives que I'on ne peut identifier a priori " (Rosental : 180, 1999) ; pour autant, le voyage semble se manifester tout à la fois comme un révélateur et un déclencheur de séjours plus longs, vécus comme des étapes dans un parcours biographique. Dans cette perspective, le récit de voyage lui-même n'est-il pas le véritable point de départ, et non le point d'arrivée seulement, du voyage ${ }^{23}$ ? II ne s'agit pas là en proposant d'interroger la place et le vécu d'un voyage dans la compréhension des parcours biographiques de mettre en évidence une causalité certainement réductrice, mais de proposer plus largement une réflexion sur le sens du mouvement et ses effets dans le renouvellement et l'élargissement des espaces vécus.

\footnotetext{
22 On peut ainsi relever le voyage mis en œuvre par le célèbre paysan polonais, le séjour parisien de Nancy Huston, celui de John Hopkins accompli au Pérou, le " voyage " autour de la chambre de Xavier de Maistre ou encore le voyage organisé du personnage décrit par Alaa El Aswany.

23 Sur cette question, on soulignera I'apport de Todorov. Celui-ci pose en effet la question de savoir si le départ de Christophe Colomb serait lié à sa lecture du récit de Marco Polo (Todorov, 1982).
} 


\section{Références bibliographiques}

\section{Ouvrages universitaires}

Barrère Anne et Martuccelli Danilo (2009) Le roman comme laboratoire. De la connaissance littéraire à l'imagination sociologique, Villeneuve-d'Ascq, Presses Universitaires du Septentrion, $373 \mathrm{p}$.

Bertaux Daniel (1976) Histoires de vies - ou récits de pratiques ? Méthodologie de l'approche biographique en sociologie, Rapport au CORDES, ronéo, 235 p.

Berthelot Jean Michel (1998) L'intelligence du social, Paris, Presses Universitaires de France, 256 p.

Berthomière William (2009) La mondialisation au prisme des migrations internationales, Mélanges de la Casa de Velázquez, 39 (1), pp. 141-160.

Bordes-Benayoun Chantal et Schnapper Dominique (2008) Les mots des diasporas, Toulouse, Presses Universitaires du Mirail, $127 \mathrm{p}$.

Certeau de Michel (1980) L'invention du quotidien, Tome 1 : Arts de faire, Paris, Gallimard, $347 \mathrm{p}$.

De Gourcy Constance (2007) Le projet migratoire entre logique d'attractivité et logique d'hospitalité. Le cas des étudiants algériens, Revue de I'Institut de Sociologie, 1 (4), pp. 131-148.

De Gourcy Constance (2005) L'autonomie dans la migration. Réflexion autour d'une énigme, préface de Michel Marié, Paris, L'Harmattan, 348 p.

Deprez Christine (2007) Langues et espaces vécus dans la migration : quelques réflexions, Langage et société, 121-122, pp. 247-257.

Detienne Marcel (2001) L'art de fonder l'autochtonie. Entre Thèbes, Athènes et le Français " de souche ", Vingtième siècle. Revue d'histoire, 69, pp. 105-110.

Diminescu Dana (2007) Le migrant connecté. Pour un manifeste épistémologique, Migrations/Société, 102, pp. 275-292.

Fouquet Thomas (2007) Imaginaires migratoires et expériences multiples de I'altérité : une dialectique actuelle du proche et du lointain, Autrepart, Paris (IRD), 41, pp. 83-97.

Habermas Jürgen (1979) Connaissance et intérêt, Paris, Gallimard, 392 p.

Ma Mung Emmanuel (2009) Le point de vue de l'autonomie dans l'étude des migrations internationales. "Penser de l'intérieur " les phénomènes de mobilité, in Françoise Dureau et Marie-Antoinette Hily Éds., Les mondes de la mobilité, Rennes, Presses Universitaires de Rennes, pp. 25-38.

Margalit Avishai (2006) L'éthique du souvenir, Climats, 218 p.

NDiaye Pap (2007) La Condition noire "Essai sur une minorité française ", préface de Marie NDiaye, Paris, Calmann-Lévy, 435 p.

Rancière Jacques (2011) Les écarts du cinéma, Paris, La Fabrique, 168 p.

Ricœur Paul (1983) Temps et récit, Tome 1 : L'Intrigue et le récit historique, Paris, Seuil, 404 p. 
Rosental Paul André (1999) Les sentiers invisibles. Espace, familles et migrations dans la France du 19e siècle, Paris, Éd. de I'EHESS, 255 p.

Said Edward (2008) Réflexions sur l'exil et autres essais, Arles, Actes Sud, 757 p.

Sayad Abdelmalek (1999) La double absence. Des illusions de l'émigré aux souffrances de l'immigré, préface de Pierre Bourdieu, Paris, Seuil, 437 p.

Sennett Richard (1995) Les Tyrannies de l'intimité, traduction d'Antoine Berman et Rebecca Folkman, Paris, Seuil, 282 p.

Teti Vito (2011) Pietre di pane. Un'antropologia del restare, Macerata, Ed. Quolibet, $192 \mathrm{p}$.

Thomas William Isaac et Znaniecki Florent (1998) Le paysan polonais en Europe et en Amérique. Récit de vie d'un migrant, Paris, Nathan, 446 p.

Timera Mahamet (2001) Les migrations des jeunes Sahéliens : affirmation de soi et émancipation, Autrepart, 18, pp. 37-49.

Todorov Tzvetan (1982) La conquête de l'Amérique. La question de l'autre, Paris, Seuil, 339 p.

Weber Max ([1947] 1964) L'éthique protestante et l'esprit du capitalisme, Paris, Plon, 285 p.

Wihtol de Wenden Catherine (2009) La globalisation humaine, Paris, Presses Universitaires de France, $288 \mathrm{p}$.

\section{Guvres littéraires}

El Aswany Alaa (2009) J'aurais voulu être Égyptien, Arles, Actes Sud, 280 p.

Hopkins John (1995) Carnets de Tanger (1962-1979), Paris, La Table Ronde, 320 p.

Huston Nancy (1999) Nord perdu, suivi de Douze France, Arles, Actes sud, 144 p.

Huston Nancy et Sebbar Leïla (1986) Lettres parisiennes. Histoires d'exil, Paris, Éd. J'ai lu, $221 \mathrm{p}$.

Maalouf Amin (1998) Les identités meurtrières, Paris, Grasset et Fasquelle, 189 p.

Maistre Xavier de ([1794] 2002) Voyage autour de ma chambre, Paris, Mille et une Nuits, 86 p.

Sebald Winfried Georg (1999) Les émigrants, Arles, Actes Sud, 308 p. 


\section{Constance De Gourcy}

\section{Partir, rester, habiter: le projet migratoire dans la littérature exilaire}

Longtemps absent des écrits sur la migration, le " projet migratoire " se présente désormais comme un outil de plus en plus mobilisé dans la littérature spécialisée, sans pour autant donner lieu à une réflexion sur ses usages. À partir d'un corpus constitué d'écrits littéraires traitant des migrations, cet article a pour objectif de proposer quelques pistes théoriques et méthodologiques permettant de préciser ses usages et de réfléchir sur ses apports. Ainsi, le recours à cette notion se révèle pertinent pour réintégrer dans I'analyse des éléments contextuels dont la portée explicative est rarement prise en compte. II devient alors possible d'envisager le parcours migratoire comme une activité dont le sens n'est pas donné a priori mais se constitue dans l'interaction avec l'environnement dans lequel évolue l'individu.

\section{Leave, Stay, Live: \\ Migration Project in the Literature of Exile}

The "migration project", which has been absent from the works on migration for a long time, now appears as a tool that is increasingly mobilized in the specialized literature. However, it doesn't provide any reflection on its uses. By analyzing a corpus of literary writings on migrations, this paper aims to propose some theoretical and methodological orientations which allow to specify its uses and to think about its contributions. Thus, it becomes relevant to use this concept in order to bring back into analysis the contextual elements whose explanatory scope is rarely taken into account. It becomes then possible to envisage the migration path as an activity whose direction is not given a priori but is being established through interaction with the environment in which the individual evolves.

\section{Irse, quedarse, vivir: el proyecto migratorio en la literatura del exilio}

El «proyecto migratorio", el cual durante mucho tiempo estuvo ausente de los escritos sobre la migración, a partir de ahora se presenta como una herramienta cada vez más movilizada en la literatura especializada. Sin embargo, no da lugar a una reflexión sobre sus usos. A partir de un corpus constituido de escritos literarios que tratan de las migraciones, este artículo tiene como objetivo proponer algunas orientaciones teóricas y metodológicas que permiten precisar sus usos y reflexionar sobre sus aportaciones. Así, resulta pertinente recurrir a esta noción para reintegrar en el análisis a los elementos contextuales cuyo alcance explicativo raramente es tomado en cuenta. Por lo tanto, se puede considerar que el trayecto migratorio es una actividad cuyo sentido no es dado a priori sino que se establece a través de la interacción del individuo con el entorno dentro del cual éste evoluciona. 